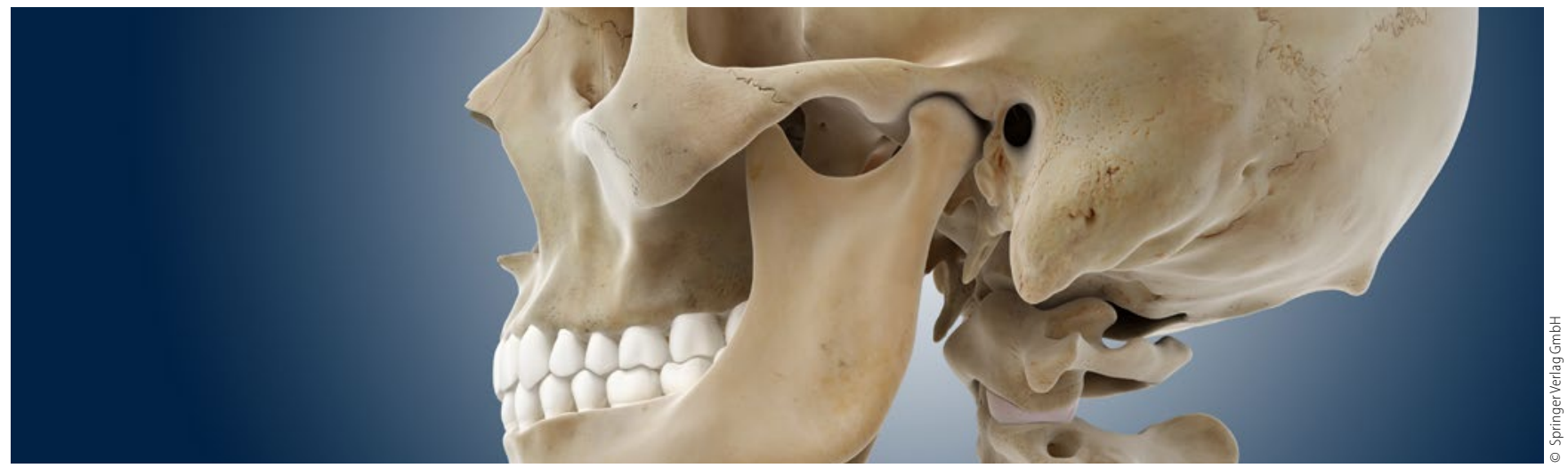

\title{
Osteomyelitis des Kiefers - eine Diagnose, einige Ursachen, viele Therapien
}

Die Osteomyelitis des Kieferknochens ist eine schwerwiegende und oft langwierige Erkrankung, die entweder akut als eitrige Entzündung auftritt oder chronisch mit einer Schwellung im Sinne einer Periostschwiele oder freiliegendem Knochen einhergeht.

Ursache kann eine bakterielle Besiedlung nach Traumata oder eine Autoimmunreaktion sein. Zudem sind iatrogen eine vorausgegangene Bestrahlung oder auch eine Therapie mit Bisphosphonaten mögliche Auslöser. So unterschiedlich wie die Ursachen, so verschieden sind auch die Therapien. Daher ist die richtige Diagnose für eine erfolgreiche Behandlung essenziell: Wird die bakteriell induzierte Osteomyelitis mit Antibiotika behandelt, so ist die immunogen verursachte Osteomyelitis mit Cortison oder gar Bisphosphonaten behandelbar, was das Krankheitsbild einer bakteriellen Osteomyelitis aggravieren würde. Glaubte man, die Osteoradionekrose sei durch eine hyperbare Sauerstofftherapie zu heilen, weiß man seit 2004, dass die Osteoradionekrose in drei Phasen entsteht:

1. Präfibrotische Phase: Entzündungsreaktion der Endothelzellen als direkte Bestrahlungsantwort.

2. Fibroblastische Phase: Atypische Fibroblasten wandern ein und führen zu einer Fehlorganisation der extrazellulären Matrix.

3. Fibroatrophe Phase: Remodelling der Matrix mit fragilem Gewebe, welches eine hohe Gefahr lokaler Bakterieninfiltration birgt.

Daher wurde auch das konservative Therapieregime der hyperbaren Sauerstofftherapie um eine medikamentös-konservative Therapie ergänzt.
Basierend auf dieser Fibroatrophietheorie hemmt die Applikation von Pentoxyphyllin (2-mal $400 \mathrm{mg}$ tgl.) und Tocopherol (1-mal $1000 \mathrm{mg}$ tgl.) über einen Zeitraum von drei Monaten synergistisch die Fibrose der Knochengefäße und die dadurch entstehende Osteoradionekrose. Bei ausbleibender klinischer Besserung kann gemäß der Autoren Lyons et al. (2008, British Journal of Oral and Maxillofacial Surgery) das Bisphosphonat Clodronat (1600 mg/Tag 5-mal pro Woche) über einen Zeitraum von weiteren drei Monaten verabreicht werden. Auch prophylaktisch vor chirurgischen Eingriffen wird die Medikation mit Pentoxyphyllin/Tocopherol empfohlen. Zudem sind eine chirurgisch-plastische Deckung und eine schonende Wundbehandlung unabdingbar. Zudem sollte perioperativ ein Antibiotikum appliziert werden.

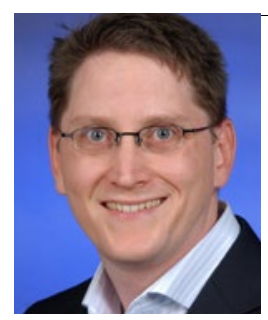

Priv.-Doz. Dr. Dr. Felix P. Koch

Autor des Wissenschaftlichen Info-Dienstes, stellvertretender Klinikdirektor und Leitender Oberarzt für Mund-, Kiefer- und Gesichtschirurgie der Uniklinik Frankfurt/Main. 